\title{
BER Improvement Using Error Concealment Technique for Pulse Code Modulation System
}

\author{
Ahmed Mohammed Ahmed \\ Assistant Lecturer, Department of Communication Engineering, Engineering College, University of \\ Diyala \\ ahmed_zydi@yahoo.com
}

\begin{abstract}
The output of pulse code modulation (PCM) encoder is statistically described; this description is used at the receiver to reduce the error of the received signal. Recently, PCM is the most important form of pulse modulation. Bit errors occur at the transmission of information through the communication system and the amount of error depends on the characteristics of the channel. In this paper, the voice message is sampled greater than Nyquist rate and quantized with scalar quantizer, then the quantized levels are encoded with resolution $\mathrm{B}$ bits assigned to each level. The binary data are mapped and transmitted over Gaussian noise channel. The system was designed without channel coding. At the receiver the statistical description is exploited to conceal the errors. The results show that a significant improvement gained in bit error rate that was reduced from 0.078 to 0.052 at $0 \mathrm{~dB}$ of $\mathrm{E}_{\mathrm{b}} / \mathrm{N}_{0}$ by soft decoding, compared to hard decoding.

Keywords: PCM, Source Coding, Hard Decoding, Soft Decoding, and MAP estimation.
\end{abstract}

Paper History: Received: (29/12/2016), Accepted: (30/4/2017)

\section{Introduction}

Digital communication means the transmission of information from the source to the destination in digital form. Essentially pulse code modulation is a method of converting an analog signal into a digital signal. The main goal of transmission information in digital form is to reduce the errors. The amount of errors in communication system depends on the signal to noise ratio $(\mathrm{S} / \mathrm{N})$. If the $\mathrm{S} / \mathrm{N}$ is established for a particular system and the rate of error is unacceptable, then some of error correction techniques must be applied.

The technique of error concealment employs the priori knowledge information about the source coding. The combination of received bit and its probability of error lead to compute the transition probability. The transition probability is used with the amount of selfprobability, number of times that the transmitted symbol is occur at the source output, to find the a posteriori probability. The MAP estimator estimates the transmitted symbol by exploiting the a posteriori probability.

Several works applied the error concealment technique to improve their systems performance's using the Channel State Information (CSI), the technique used different amount of priori knowledge with the fading channel, Soft Output Viterbi Algorithm was used to compute the transition probabilities [1]. Driven framework provide for variable length soft decoding and several conditions were discussed for variable length and fixed length soft decoding in point of view of performance tradeoffs [2]. Three techniques used softdecision audio decoding to improve the quality of audio and reducing the decoding delay, these three techniques depend on the serial predictor cascades, forward-backward prediction, and exploiting the explicit redundancy [3]. Exploiting the priori knowledge about the transmitted parameter levels, is new concept presented, and the convolutional decoding is aided by soft decision decoding [4].

In this paper, the voice message is sampled greater than Nyquist rate and quantized with scalar quantizer. The output of the quantizer is encoded with fixed length source coding, with resolution $B$, where $B$ is the number of bits assigned for each transmitted parameter or level. The output of PCM encoder statistically described, that means self-probability was obtained for each parameter, and exploit this description at receiver to find the a posteriori probability [5]. The code words were mapped and transmitted over Gaussian noise channel. At the receiver the combination of selfprobability and transition probability is exploited to find the a posteriori probability, the transmitted parameters are estimated by a maximum a posteriori probability in the last block in the error concealment.

\section{The Proposed System Description}

The overall transmission system is depicted in Figure 1 . The voice message $\widetilde{v}$ which is band 
limited to $8 \mathrm{KHz}$ was sampled with $35 \%$ greater than the Nyquist rate. Selecting high sampling rate value to gives more accurate statistical description at the output of PCM encoder. The series of samples which varies between the minimum and maximum value of input voice signal are quantized with scalar quantizer. The output of the scalar quantizer is a sequence of numbers of $x$, where $x=$ $\left[x_{0}, x_{1}, x_{2}, \ldots x_{i}\right], \quad x_{i} \in Q t \quad$ with $i=$ $\left[0,1,2, \ldots, 2^{B}-1\right]$. The outputs of the quantizer are encoded with $B$ bit assign for each quantization levels; the resolution of coded parameters is selected to be 5 bits .

The self-probability for each symbol of the PCM encoder outputs is statistically computed. The self-probabilities $p\left(x_{i}\right)$ that represent the number of times that the symbol occurs at the output of the PCM encoder are saved as a vector at the receiver side considering a priori knowledge about the source encoder. In this PCM system, zero priori knowledge is used to find the a posteriori probabilities. In addition to the statistical description of the output of the source encoder, the sequences of bits are converted to a digital signal using mapping technique. So the digital data is converted to bipolar signal of a form $x_{0}^{b} \in\{-1,+1\}$.

Bipolar signal is transmitted over Gaussian channel using baseband transmission, where a white Gaussian noise is added to the transmitted digital signal over the channel. In Gaussian channel no amplitude loss (fading does not exist) distortion is introduced by the noise [6].

Transition probabilities are computed by exploiting the $L_{c}$ which represent the channel state information (CSI), and can be found by:

$$
L_{c}=4 * \frac{E_{b}}{N_{0}}
$$

Where $E_{b}$ energy of transmitted bit, and $N_{0}$ is white noise [1].

Then, the bit error probability of the hard decided bit can be formulated as [7]

$$
p_{e 0}(b)=\frac{1}{1+\exp \left|L_{c} * y_{0}(b)\right|}
$$

The received combination of hard bit $y_{0}(b)$ and its instantaneous bit error probability $p_{e 0}(b)$ is named soft bit. From the instantaneous bit error probability, the conditional bit probability for transition of transmitted bit to the known received bit can be computed using [5]

$$
\begin{gathered}
p\left(y_{0}(b) / x_{0}^{i}(b)\right)= \\
\left\{\begin{array}{c}
1-p_{e 0}(b) \quad \text { if } y_{0}(b)=x_{0}^{i}(b) \\
p_{e 0}(b) \quad \text { if } y_{0}(b) \neq x_{0}^{i}(b)
\end{array}\right.
\end{gathered}
$$

The channel is memoryless channel, so the codeword transition probability is given by[5]:

$$
p\left(y_{0} / x_{0}^{i}\right)=\prod_{b=1}^{B} p\left(y_{0}(b) / x_{0}^{i}(b)\right)
$$

The a posteriori probabilities for the transmitted symbols are computed using self-probability for the symbol and the transition probability as described by Bayes rule [8].

$$
p\left(x_{0}^{i} / y_{0}\right)=\frac{p\left(y_{0} / x_{0}^{i}\right) p\left(x_{0}^{i}\right)}{\sum_{i=0}^{2^{B}-1} p\left(y_{0} / x_{0}^{i}\right) p\left(x_{0}^{i}\right)}
$$

Maximum a posteriori probability estimator is used to estimate the transmitted encoded symbol; MAP estimator is selected because it minimizes the probability error for the decoded symbol. Decoded symbols in MAP represent single codeword from the quantization Table [5].

$$
\hat{v}=\max _{i} p\left(x_{0}^{i} / y_{0}\right)
$$

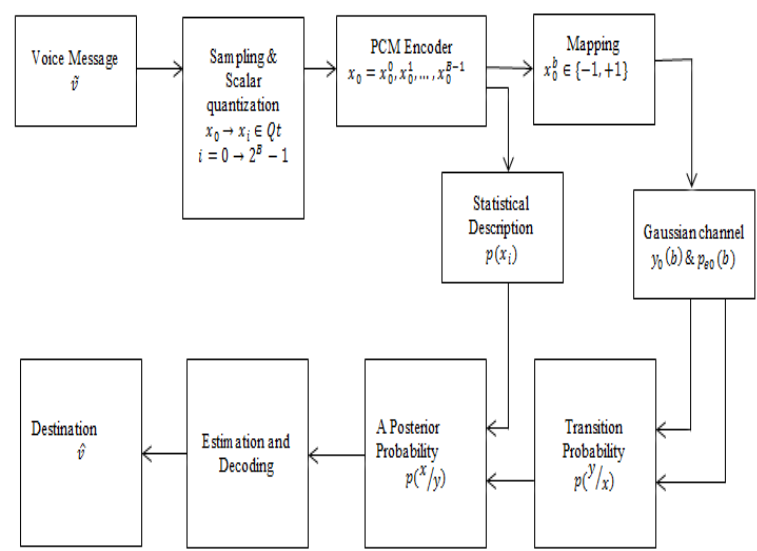

Figure 1: Block diagram of proposed PCM communication system

\section{Results and Discussion}

The BER is considered as a key that is used for evaluating the digital communication systems. Performance of the proposed PCM communication system which is depicted in Figure 1 is evaluated in term of Bit Error Rate (BER). The voice message $\widetilde{v}$ which is band limited to $8 \mathrm{KHz}$ sampled with $35 \%$ greater than the Nyquist rate. Then the discrete signal is quantized with scalar quantizer with resolution of 5 bits assigned to each parameter level. The series of binary data are mapped to $\{-1,+1\}$, and transmitted over Gaussian channel.

As shown in Figure 2, the performance of the proposed PCM system in term of BER, that the 
theoretical BER and simulated BER are identical, this is due to that the system is simulated without any knowledge about the behavior of source encoder output.

The performance of the proposed PCM system using priori knowledge is better than the system without priori knowledge as shown in Figure 3, remarkable BER improvement obtained of about 0.0264 at $0 \mathrm{~dB}$ of $E_{b} / N_{0}$ by exploiting the behavior (statistical description) of the source encoder. The statistical description of the source encoder output assists the receiver to conceal the error. The amount of error concealment depends on the amount of the priori knowledge obtained about the output of source encoder.

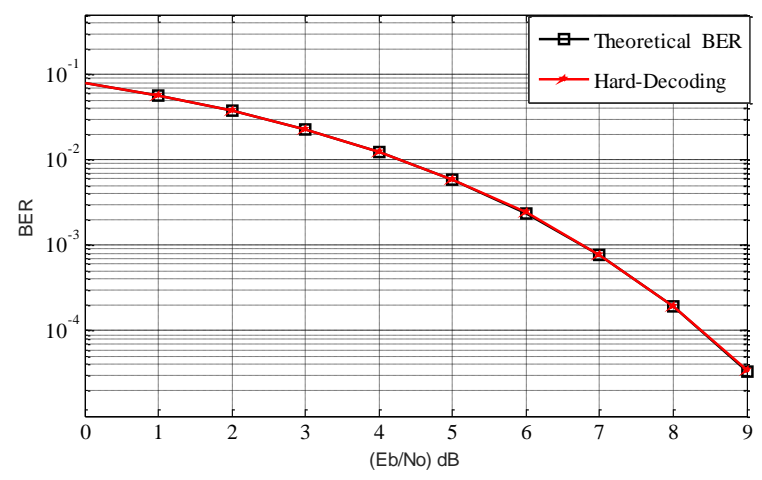

Figure 2: BER Performance for Theoretical and

Hard Decoding

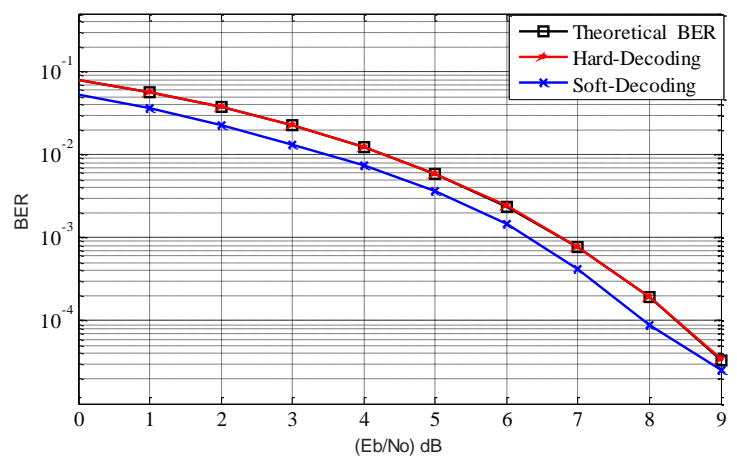

Figure 3: BER Performance for Hard Decoding and Soft Decoding

\section{Conclusion}

By using MATLAB software, the Pulse code modulation system which is depicted in Figure 1 was simulated. The system was implemented without channel coding (error correction techniques).The Performance of the system is evaluated in terms of BER and the results show that a significant amount of BER improvement obtained of about 0.0264 at $0 \mathrm{~dB}$ of $\mathrm{E}_{\mathrm{b}} / \mathrm{N}_{0}$ by exploit the knowledge about the output of source encoder, the amount of improvement depends on the amount of the priori knowledge. The results also show that, there is trade of between the value of resolution and the number of samples applied to the system, huge number gives more accurate statistical description.

\section{References}

[1]. Fingscheidt, Tim, and Peter Vary. Robust speech decoding: A universal approach to bit error concealment, Acoustics, Speech, and Signal Processing, 1997. ICASSP-97., 1997 IEEE International Conference on. Vol. 3. IEEE, 1997.

[2]. Han, Sai, and Tim Fingscheidt. Variablelength versus fixed-length coding: On tradeoffs for soft-decision decoding. 2014 IEEE International Conference on Acoustics, Speech and Signal Processing (ICASSP). IEEE, 2014. [3]. Pflug, Florian, and Tim Fingscheidt. "Robust ultra-low latency soft-decision decoding of linear PCM audio." IEEE Transactions on Audio, Speech, and Language Processing 21.11 (2013): 2324-2336.

[4] Heinen, Stefan, Axel Geiler, and Peter Vary. "MAP channel decoding by exploiting multilevel source a priori knowledge." ITG FACHBERICHT (1998): 89-94.

[5] Fingscheidt, Tim, and Peter Vary. Softbit speech decoding: A new approach to error concealment. IEEE Transactions on Speech and Audio Processing 9.3 (2001): 240-251.

[6] Xiong. Fuqin. ARTECH HOUSE, INC. Digital Modulation Techniques. pp 4. Artech House. Boston. London, 2000.

[7] J. Hagenauer, Viterbi decoding of convolutional codes for fadingand burstchannels, in Proc. 1980 Zurich Seminar Digital Commun., Zürich, Switzerland, 1980, pp. G2.1-G2.7. Hagenauer, Joachim. "Sourcecontrolled channel decoding." IEEE Transactions on Communications 43.9 (1995), 2449-2457.

[8] Farrell, Patrick Guy. Essentials of errorcontrol coding. John Wiley \& Sons, 2006. 